\title{
Relationship between impulse oscillometry system and conventional lung function test for diagnosis of bronchiolitis obliterans in children
}

Hye Jin Lee ${ }^{1}$, Kyunghoon $\mathrm{Kim}^{2}$, Seong koo Kim ${ }^{1}$, Jae Wook Lee ${ }^{1}$, Jong-Seo Yoon ${ }^{2}$, Nack Gyun Chung ${ }^{1}$, and Bin Cho ${ }^{1}$

${ }^{1}$ Catholic University of Korea College of Medicine

${ }^{2}$ Catholic University of Korea Eunpyeong St Mary's Hospital

February 9, 2021

\begin{abstract}
Background: Functional assessment of small airways in young children with bronchiolitis obliterans (BO) is challenging due to their relative inaccessibility and the generally poor lung function test performance of these patients. We analyze the correlation between impulse oscillometry (IOS), spirometry, and plethysmographic parameters in pediatric BO patients. Methods: A total of 89 IOS assessments of pediatric BO patients or children without lung disease were included, and the relationship between pulmonary function tests (PFTs) and diagnostic performance were analyzed. Results: R5, R5-20, X5, and AX were statistically significantly worse in the BO group. In Spearman's correlation analysis, AX and R5 showed the strongest correlation with conventional PFT parameters, and AX was the variable with the highest relative correlation with FEV1, FEF25-75\%, and both measures of plethysmographic resistance. Receiver operating curve analysis highlighted AX and Raw\% pred as the most optimal parameters for $\mathrm{BO}$ diagnostic performance with areas under curve of 0.811 and 0.827 , respectively. Conclusion: The AX and R5 parameters can be useful in identifying the severity of airway obstruction in children with BO, and IOS more generally can accurately detect pathological obliteration of small airways in pediatric BO patients.
\end{abstract}

\section{ORIGINAL ARTICLE}

Relationship between impulse oscillometry system and conventional lung function test for diagnosis of bronchiolitis obliterans in children

Hye Jin Lee, MD, Kyunghoon Kim, MD, PhD, Seong koo Kim, MD, Jae Wook Lee, MD, PhD, Jong-seo Yoon, MD, PhD, Nack-Gyun Chung, MD, PhD, Bin Cho, MD, PhD

Department of Pediatrics, College of Medicine, The Catholic University of Korea, Seoul, Republic of Korea Correspondence Professor Jong-seo Yoon, MD, PhD

Division of Respiratory and Allergy, Department of Pediatrics, College of Medicine, The Catholic University of Korea, 222, Banpo-daero, Seocho-gu, Seoul, 06591, Republic of Korea

Tel: +82-2-2258-6190, Fax: +82-2-537-4544, E-mail:pedjsyoon@catholic.ac.kr

ORCID : 0000-0002-3785-463

KEYWORDS

Bronchiolitis obliterans, impulse oscillometry system, lung function, children

Running head: 
Impulse oscillometry for the diagnosis of bronchiolitis obliterans in children

\begin{abstract}
Background: Functional assessment of small airways in young children with bronchiolitis obliterans (BO) is challenging due to their relative inaccessibility and the generally poor lung function test performance of these patients. We analyze the correlation between impulse oscillometry (IOS), spirometry, and plethysmographic parameters in pediatric $\mathrm{BO}$ patients.
\end{abstract}

Methods: A total of 89 IOS assessments of pediatric BO patients or children without lung disease were included, and the relationship between pulmonary function tests (PFTs) and diagnostic performance were analyzed.

Results: R5, R5-20, X5, and AX were statistically significantly worse in the BO group. In Spearman's correlation analysis, AX and R5 showed the strongest correlation with conventional PFT parameters, and AX was the variable with the highest relative correlation with FEV1, FEF25-75\%, and both measures of plethysmographic resistance. Receiver operating curve analysis highlighted AX and Raw\% pred as the most optimal parameters for BO diagnostic performance with areas under curve of 0.811 and 0.827 , respectively.

Conclusion: The AX and R5 parameters can be useful in identifying the severity of airway obstruction in children with BO, and IOS more generally can accurately detect pathological obliteration of small airways in pediatric $\mathrm{BO}$ patients.

\title{
1 INTRODUCTION
}

Bronchiolitis obliterans (BO) is a fibrosing chronic obstructive lung disease that leads to the obliteration of the small airways. ${ }^{1} \mathrm{BO}$ occurs secondarily to several conditions including infection, allogeneic hematopoietic stem cell transplantation (HSCT), and lung transplants. ${ }^{2-4}$ Pulmonary function tests (PFTs) are essential for the diagnosis and management of $\mathrm{BO}$, and spirometry is considered the gold standard for determining the severity of obstructive pulmonary disease. However, the accurate assessment of the degree of airway obliteration in young children with BO is challenging due to the relative inaccessibility of the small airways and the complexity of the distal lung lesions involved.

Compared to spirometry, impulse oscillometry (IOS) is a simpler and less invasive method of assessing small to total airway resistance in children. ${ }^{5}$ Recent studies have shown that IOS is capable of quantifying the degree of small airway impairments. ${ }^{6-8}$ The correlation between IOS and lung function as measured by spirometers has already been evaluated in children with asthma,,${ }^{7,9-12}$ cystic fibrosis, ${ }^{13,14}$ and other chronic obstructive lung disease. ${ }^{15,16}$ Irreversible fibrosis and obstruction are the major pathologic components of $\mathrm{BO}$ that distinguishes it from other chronic obstructive lung diseases, but studies of using IOS with pediatric BO patients are limited. In this study, we analyze the correlations between IOS, spirometry, and body plethysmography in children with BO and suggest how IOS parameters reflect the conventional PFT variables that physicians are familiar with.

\section{SUBJECTS AND METHODS}

\subsection{Study Population}

We conducted a retrospective chart review of patients who underwent IOS assessment between March 2018 and February 2019 at the Department of Pediatrics at Seoul St. Mary's Hospital in the College of Medicine at the Catholic University of Korea. Inclusion criteria were (a) being under 18 years of age; (b) physiciandiagnosed BO; and (c) the ability to perform IOS. Patients were excluded from the study if they had asthma or an obstructive lung disease, and healthy children were enrolled as a normal control group. Any patients who had undergone IOS for routine lung function assessment prior to stem cell transplantation in Department of hemato-oncology were also included in the controls. IOS, spirometry, and/or body plethysmography were repeated at outpatient visits with written informed consent obtained from the parent or guardian of each participant. 


\subsection{IOS and PFTs}

All IOS procedures were performed using Tremoflo (Thorasys Inc., Montreal, QC) with measurements made according to the standards adopted by the European Respiratory Society. ${ }^{17}$ IOS was performed with the child sitting upright, a clip to block the nose, lips sealed around the mouthpiece, and cheeks supported with the hands to decrease dead space. The mean values of at least two recordings that met the criteria of $>$ 0.80 coherence were used for analysis. The IOS parameters R5, R20, R5-20, AX, and X5 were measured. Other PFTs were performed with a Vmax instrument (Sensor Medics, VIASYS Healthcare, Yorba Linda, CA) according to guidelines from the American Thoracic Society and European Respiratory Society.

\subsection{Statistical Analysis}

The categorical and continuous variables were compared using the $\chi^{2}$ and Student's t-test, respectively. The IOS and PFT parameters were analyzed using Spearman's Rank correlation given that they were not normally distributed, and multivariate logistic and linear regression analysis using the IOS parameters and PFT values was performed to analyze correlations between variables. The receiver operating characteristic (ROC) method was applied to evaluate the utility of different IOS parameters for predicting physiciandiagnosed BO. Areas under the curve (AUCs) and optimal cut-off points based on maximizing the sum of sensitivity and specificity were calculated for each IOS parameter. $\mathrm{P}$ values of $<0.05$ were considered statistically significant, and the statistical analyses were performed using R Version 3.6.2 (https://cran.rproject.org/web/packages/maxstat/index.html). This study was approved by the Institutional Review Board of Seoul Saint Mary's hospital (number: KC21RISI0028).

\section{RESULTS}

\subsection{Patient Characteristics}

The demographic and clinical characteristics of the patients are presented in Table 1. 89 assessment of IOS were performed in 41 participants, including 15 normal controls and $26 \mathrm{BO}$ patients. Of the $26 \mathrm{BO}$ patients, $20(76.9 \%)$ had previously undergone HSCT. Spirometry and body plethysmography were performed in 43 $(48.3 \%)$ and $29(32.6 \%)$ cases, respectively, with failed instances including patients who were too young or unable to take forced inspiration and expiration due to severe BO. The mean ages of the control and BO groups was 7.7 and 12.5 years, respectively. In the PFTs, FVC, FEV1, FEV1/FVC, FEF25-75\%, and RV were statistically significantly worse in the BO group. Raw\% ( $83.4 \pm 36.6$ vs $263.6 \pm 234.1 ; \mathrm{p}=0.006)$ and sRaw\% (114.1 \pm 46.4 vs $463.1 \pm 488.2 ; \mathrm{p}=0.008)$ were significantly higher. Among the IOS parameters, R5-20, AX, X5, R5, and AX values were all statistically significantly worse in the BO group (Table 1).

\subsection{Linear Correlation}

In Spearman's Rank analysis, the AX and R5 IOS parameters showed the strongest correlation with conventional PFTs (Table 2). Specifically, the AX Z-score showed a statistically significant correlation with FEV1 $(\mathrm{r}=-0.84, \mathrm{p}=0.001)$, FEV1/FVC $(\mathrm{r}=-0.87, \mathrm{p}<0.001)$, FEF25-75\% $(\mathrm{r}=-0.94, \mathrm{p}<0.001)$, and Raw\% ( $\mathrm{r}$ $=0.66, \mathrm{p}=0.004)$. The R5 Z-score showed strong negative correlations with these spirometry parameters.

The multivariate linear regression analysis showed that most IOS parameters had a statistically significant correlation with conventional airway obstruction and resistance values; FVC, FEV1, FEV1/FVC, FEF25$75 \%$, Raw, and sRaw (Supplement 1). Figure 1 illustrates the relative prediction weights of the IOS parameters for obstructive PFTs using backward elimination analysis. The AX parameter had the highest relative correlation in FEV1 and FEF25-75\%, and in both measures of plethysmographic resistance, namely Raw and sRaw (Figure 1).

\subsection{ROC and Logistic Regression}

In ROC analysis, the optimal cut-off values of each IOS and plethysmographic parameter for BO diagnosis were obtained (Figure 2), and the AUCs and sensitivity-specificity for each cut-off are presented in Table 3. The predictive reference values of Raw and sRaw have not been established in children, and so we used 
$103 \%$ and $203.5 \%$ as the respective cut-off values following ROC analysis. The representative variables that showed the most optimal BO diagnostic performance were AX and Raw\% (AUC; 0.811 vs 0.827).

Multivariate logistic regression analysis obtained the odds ratios (ORs) of the IOS parameters for PFT and plethysmographic measurements, divided into two groups: normal and obstructive (Table 4). The criteria for the obstructive group were FEV1 and FEV1/FVC of 75 and a FEF25-75\% of 60 . Most of the IOS parameters relating to resistance and reactance showed statistically significant correlations with FEV1, FEV1/FVC, FEF25-75\%, Raw, and sRaw. The R5 Z-score showed the highest ORs with FEV1, FEF25-75\%, Raw, and sRaw (OR; 3.94, 5.96, 4.88, and 9.94, P-v values; 0.006, 0.005, 0.04, and 0.032, respectively).

\section{DISCUSSION}

IOS is a method of assessment based on forced vibration technology which has the advantage particularly for children, of being relatively effort independent. ${ }^{18,19}$ IOS can be used to measure respiratory resistance by applying small pressures to the mouth which are transmitted to the lungs. Our study showed that the proportion of children who performed IOS was much higher than that of spirometry or plethysmography. Through IOS, lung function evaluation was possible particularly in young children, who had difficulty understanding and cooperating with conventional PFT performance.

In the correlation analysis between IOS and spirometry parameters, strong linear correlations were identified for R5 and AX. These parameters refer to resistance and reactance at different locations from the central to the peripheral airway. Resistance is a component of lung impedance and provides information about the forward pressure of the airway, and reactance is the repulsive resistance of distensible airways by area. Since the low oscillation frequencies of the IOS can be transmitted more distantly than the higher frequencies, resistance at $5 \mathrm{~Hz}(\mathrm{R} 5)$ represents peripheral and total airway resistance, and resistance at 20 $\mathrm{Hz}$ (R20) represents the large airways. ${ }^{20-22}$ It is well established that changes in resistance become evident at low frequencies in patients with peripheral airway disease. ${ }^{21}$ Small airway resistance can be calculated by subtracting R20 from R5, and can be used with AX and X5 to reflect changes in the degree of obstruction in peripheral airways. ${ }^{22,23}$

Our results showed that AX was the most important parameter in a linear relationship with the obstructive PFT variables, and had a particularly high AUC value for BO diagnosis in ROC analysis. AX, a reactance parameter, represents the area under the curve which is the total reactance at all frequencies between $5 \mathrm{~Hz}$ and Fres. ${ }^{21} \mathrm{AX}$ therefore reflects the elastic properties of the lungs in all frequency domains and also provides information on peripheral airway obstruction. The deterioration of the peripheral airway tissue in terms of changes in compliance and elasticity due to fibrosis and obliteration in BO patients can be better represented by this overall value rather than resistance or reactance at specific frequencies. There have been IOS studies of a range of diseases, but it has so far been best characterized for use with asthma patients. People with asthma have been shown to exhibit more negative R5, AX, Fres, and X5 compared to controls. ${ }^{20,23-25} \mathrm{~A}$ sensitivity cut-off in AX and R5 has been suggested to detect bronchodilator responses ${ }^{19,26,27}$ and control of asthma. ${ }^{6,21,25}$ Our study supports the possibility that AX is the most sensitive indicator of airway obstruction and resistance in $\mathrm{BO}$ patients specifically.

R5 Z-score showed the most statistically significant OR for airway resistance of the plethysmography in this study. In contrast to most of the IOS parameters represented high OR values for the obstructive spirometry variables; FEV1, FEV1/FVC, and FEF25-75\%, only specific IOS parameters with respect to plethysmographic resistance showed statistically significant results. These results represent the three-dimensional anatomical relationship between resistance or reactance obtained through IOS by tidal breathing, through spirometry using forced exhalation, and through body plethysmography. Raw\% and sRaw\% also showed good AUC in ROC analysis for the diagnosis of BO. Specific airway resistance, sRaw, can be measured plethysmographically through the simultaneous measurement of air flow and volume swing during tidal breathing and is applicable to children over two years of age. This measure provides an estimate of resistance that is practically independent of body size or age beyond infancy being the product of Raw and thoracic gas volume. ${ }^{28,29}$ It reflects the overall dimensions of the airway, including the effect of lung expansion on its 
caliber, and so it is a more dynamic parameter compared to Raw. ${ }^{29,30}$ Raw itself has been reported to be more sensitive to detecting obstruction in the extra thoracic or large central airways than in peripheral system. ${ }^{30,31}$ Our findings indicated that plethysmographic resistance showed excellent correlation with the IOS parameters relating to peripheral airway resistance such as AX and R5.

Our study has two notable limitations: (1) The sample size was small, and the majority of the BO patients had undergone HSCT, so that presentation of different pulmonary function characteristics was limited. The BO group was heterogeneous in terms of pathophysiological status and severity at the time of IOS; (2) In those control cases where IOS was performed before HSCT, previous immunosuppressive therapy may have affected lung function. Despite these limitations, our study has the strengths of reporting IOS in BO patients, where there is otherwise insufficient research evidence, and approaching various PFTs in multiple ways to analyze the relationship from various perspectives.

In conclusion, the resistance and reactance IOS parameters were significantly poor in the BO group and showed a high correlation with other obstructive PFTs. IOS is a method that can accurately detect pathological small airway obstruction in pediatric BO patients, independent of conventional PFT. The measurement of AX and R5 are easy, and useful in identifying the severity of airway obstruction in children with BO.

\section{Author Contributions}

Jong-seo Yoon conceived the research idea. Kyunghoon Kim, Seongkoo Kim, Jae Wook Lee, Jong-seo Yoon, Nack Gyun Chung, and Bin Cho were responsible for the diagnosis and treatment of the patients and collected the clinical and laboratory data. All authors reviewed and approved the final version.

\section{REFERENCES}

1. Kavaliunaite E, Aurora P. Diagnosing and managing bronchiolitis obliterans in children. Expert Rev Respir Med.2019;13(5):481-488.

2. Williams KM. How I treat bronchiolitis obliterans syndrome after hematopoietic stem cell transplantation. Blood.2017;129(4):448-455.

3. Kurland G, Michelson P. Bronchiolitis obliterans in children.Pediatr Pulmonol. 2005;39(3):193-208.

4. Champs NS, Lasmar LMLBF, Camargos PAM, Marguet C, Fischer GB, Mocelin HT. Post-infectious bronchiolitis obliterans in children. J Pediatr (Rio J). 2011;87(3):187-198.

5. Komarow HD, Myles IA, Uzzaman A, Metcalfe DD. Impulse oscillometry in the evaluation of diseases of the airways in children. Ann Allergy Asthma Immunol. 2011;106(3):191-199.

6. Zheng S, Hu Y, Chen Z, Wang M, Liao W. Predicting asthma exacerbation by impulse oscillometry evaluation of small airway function and fractional exhaled nitric oxide in preschool children. Pediatr Pulmonol. 2020;55(7):1601-1607.

7. Mondal P, Yirinec A, Midya V, et al. Diagnostic value of spirometry vs impulse oscillometry: A comparative study in children with sickle cell disease. Pediatr Pulmonol. 2019;54(9):1422-1430.

8. Lundblad LKA, Miletic R, Piitulainen E, Wollmer P. Oscillometry in Chronic Obstructive Lung Disease: In vitro and in vivo evaluation of the impulse oscillometry and tremoflo devices. Sci Rep.2019;9(1):11618.

9. Batmaz SB, Kuyucu S, Arıkoglu T, Tezol O, Aydogdu A. Impulse oscillometry in acute and stable asthmatic children: a comparison with spirometry. J Asthma. 2016;53(2):179-186.

10. Jara-Gutierrez P, Aguado E, Del Potro MG, Fernandez-Nieto M, Mahillo I, Sastre J. Comparison of impulse oscillometry and spirometry for detection of airway hyperresponsiveness to methacholine, mannitol, and eucapnic voluntary hyperventilation in children. Pediatr Pulmonol. 2019;54(8):1162-1172.

11. Sugawara H, Saito A, Yokoyama S, Tsunematsu K, Chiba H, Takahashi H. A retrospective analysis of usefulness of impulse oscillometry system in the treatment of asthma. Respir Res. 2020;21(1):226. 
12. McDowell KM. Recent Diagnosis Techniques in Pediatric Asthma: Impulse Oscillometry in Preschool Asthma and Use of Exhaled Nitric Oxide. Immunol Allergy Clin North Am. 2019;39(2):205-219.

13. Moreau L, Crenesse D, Berthier F, Albertini M. Relationship between impulse oscillometry and spirometric indices in cystic fibrosis children. Acta Paediatr. 2009;98(6):1019-1023.

14. Buchs C, Coutier L, Vrielynck S, Jubin V, Mainguy C, Reix P. An impulse oscillometry system is less efficient than spirometry in tracking lung function improvements after intravenous antibiotic therapy in pediatric patients with cystic fibrosis. Pediatr Pulmonol.2015;50(11):1073-1081.

15. Björn L, Erik M, Per T, Mikael N, Jenny H. Agreement between spirometry and impulse oscillometry for lung function assessment in 6-year-old children born extremely preterm and at term. Pediatr Pulmonol. 2020;55(10):2745-2753.

16. Lauhkonen E, Riikonen R, Törmänen S, et al. Impulse oscillometry at preschool age is a strong predictor of lung function by flow-volume spirometry in adolescence. Pediatr Pulmonol. 2018;53(5):552-558.

17. Oostveen E, MacLeod D, Lorino H, et al. The forced oscillation technique in clinical practice: methodology, recommendations and future developments. Eur Respir J. 2003;22(6):1026-1041.

18. Cogswell JJ. Forced oscillation technique for determination of resistance to breathing in children. Arch Dis Child.1973;48(4):259-266.

19. Park JH, Lee JH, Kim HJ, et al. Usefulness of impulse oscillometry for the assessment of bronchodilator response in elderly patients with chronic obstructive airway disease. J Thorac Dis.2019;11(4):1485-1494.

20. Bickel S, Popler J, Lesnick B, Eid N. Impulse oscillometry: interpretation and practical applications. Chest.2014;146(3):841-847.

21. Shi Y, Aledia AS, Tatavoosian AV, Vijayalakshmi S, Galant SP, George SC. Relating small airways to asthma control by using impulse oscillometry in children. J Allergy Clin Immunol.2012;129(3):671-678.

22. Brashier B, Salvi S. Measuring lung function using sound waves: role of the forced oscillation technique and impulse oscillometry system. Breathe (Sheff). 2015;11(1):57-65.

23. Shin YH, Yoon JW, Choi SH, et al. Use of impulse oscillometry system in assessment of asthma severity for preschool children. J Asthma. 2013;50(2):198-203.

24. Sheen YH, Jee HM, Ha EK, et al. Impulse oscillometry and spirometry exhibit different features of lung function in bronchodilation. J Asthma. 2018;55(12):1343-1351.

25. Lee JY, Seo JH, Kim HY, et al. Reference values of impulse oscillometry and its utility in the diagnosis of asthma in young Korean children. $J$ Asthma. 2012;49(8):811-816.

26. Komarow HD, Skinner J, Young M, et al. A study of the use of impulse oscillometry in the evaluation of children with asthma: analysis of lung parameters, order effect, and utility compared with spirometry.Pediatr Pulmonol. 2012;47(1):18-26.

27. Olaguíbel JM, Alvarez-Puebla MJ, Anda M, et al. Comparative analysis of the bronchodilator response measured by impulse oscillometry (IOS), spirometry and body plethysmography in asthmatic children. $J$ Investig Allergol Clin Immunol. 2005;15(2):102-106.

28. Klug B, Bisgaard H. Specific airway resistance, interrupter resistance, and respiratory impedance in healthy children aged 2-7 years. Pediatr Pulmonol. 1998;25(5):322-331.

29. Buhr W, Jörres R, Knapp M, Berdel D. Diagnostic value of body plethysmographic parameters in healthy and asthmatic young children is not influenced by breathing frequency. Pediatr Pulmonol.1990;8(1):23-28.

30. Bisgaard H, Nielsen KG. Plethysmographic measurements of specific airway resistance in young children. Chest. 2005;128(1):355-362. 
31. Mauger-Hamel P, Du Boisbaudry C, Léon K, Alavi Z, Giroux-Metges MA. Relationship between baseline and post-bronchodilator interrupter resistance and specific airway resistance in preschool children.Ann Allergy Asthma Immunol. 2020;124(4):366-372.

\section{Image Legends}

FIGURE 1 Relative importance as predictors of IOS variables for obstructive PFT parameters; (A) FEV1 (B) FEF $25-75 \%$ (C) Raw\% (D) sRaw\%

FIGURE 2 Receiver operating characteristic curve showing sensitivity and 1-specificity of IOS parameters for the diagnosis $\mathrm{BO}$ with (A) R5-20, X5 cmH2O/L/s and AX cmH2O/L (B) R5 and AX z-score

\section{Hosted file}

TABLE.pdf available at https://authorea.com/users/394665/articles/508038-relationshipbetween-impulse-oscillometry-system-and-conventional-lung-function-test-for-diagnosisof-bronchiolitis-obliterans-in-children

(A)

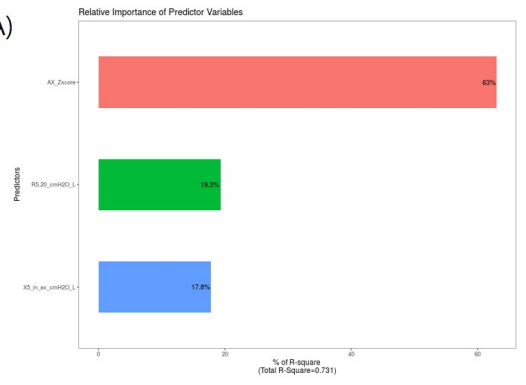

(C)

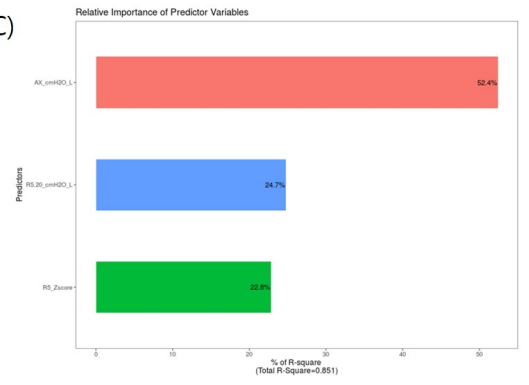

(A)

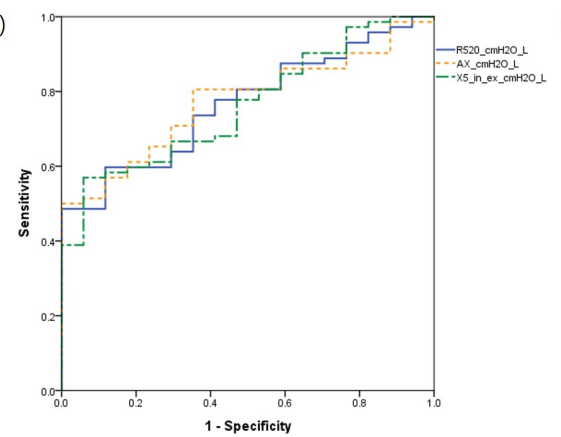

(B)

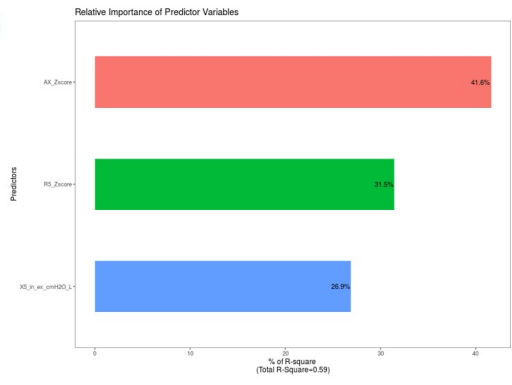

(D)

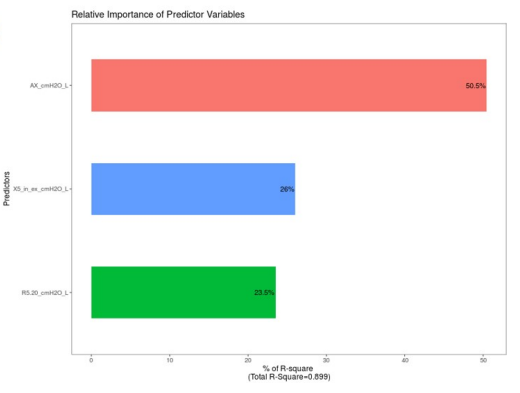

(B)

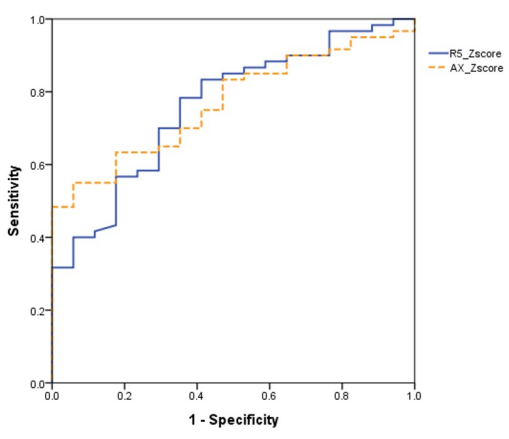

